\title{
Bridging the Gap in Neurotherapeutic Discovery and Development: The Role of the National Institute of Neurological Disorders and Stroke in Translational Neuroscience
}

\author{
Meghan Mott $^{1} \cdot$ Walter Koroshetz $^{1}$
}

Published online: 17 June 2015

(C) American Astronautical Society (Outside the U.S.) 2015

\begin{abstract}
The mission of the National Institute of Neurological Disorders and Stroke (NINDS) is to seek fundamental knowledge about the brain and nervous system and to use that knowledge to reduce the burden of neurological disease. NINDS supports early- and late-stage therapy development funding programs to accelerate preclinical discovery and the development of new therapeutic interventions for neurological disorders. The NINDS Office of Translational Research facilitates and funds the movement of discoveries from the laboratory to patients. Its grantees include academics, often with partnerships with the private sector, as well as small businesses, which, by Congressional mandate, receive $>3 \%$ of the NINDS budget for small business innovation research. This article provides an overview of NINDS-funded therapy development programs offered by the NINDS Office of Translational Research.
\end{abstract}

Keywords Therapy development $\cdot$ Translational neuroscience $\cdot$ Preclinical discovery $\cdot$ Neurological health

More than 600 disorders afflict the nervous system, striking an estimated 50 million Americans each year. Some of these disorders are well known, including stroke, epilepsy, Parkinson's disease, and autism, but many others are rare and devastating, known only by the families affected and the doctors who treat them.

Meghan Mott

mottmc@od.nih.gov

1 National Institute of Neurological Disorders and Stroke, National Institutes of Health, Bethesda, MD, USA
This year marks the 65th anniversary of the creation of the National Institute of Neurological Disorders and Stroke (NINDS) at the National Institutes of Health (NIH). Our mission at NINDS is to seek fundamental knowledge about the brain and nervous system and to use that knowledge to reduce the burden of neurological disease. This mission was recently revised to reflect our commitment to basic neuroscience research, where NINDS has historically served as the largest single funder [1]. With an annual budget of $\$ 1.6$ billion, NINDS supports the taxpayers' investment in neurological health by funding the best science across the full spectrum of basic, translational, and clinical research.

Our commitment to research into understanding the fundamental neural mechanisms of normal and abnormal structure and activity of the human nervous system forms the base for translating discoveries into new therapies. NINDS-funded research plays a central role in understanding pathogenic mechanisms underlying neurological disorders. It also plays a central role in describing their natural history, underlying risk factors, whether these are genetic or environmental. The role of NINDS in the process of therapy development is more controversial and in flux.

Many of industry's translational neuroscience programs shrunk or closed in the last decade as central nervous system disorders acquired the reputation of being too complicated and too risky for investors and industry [2]. The challenges of unknown mechanisms of disease, lack of predictive animal models, lack of useful biomarkers of disease progression, particularly long clinical development and approval times, and low clinical approval success rates are seen as inherent risks. In addition, the economic downturn reduced the available capital for smaller biotechnology firms that were so important for linking the discoveries from academia to industry. The most glaring disconnect between public neurologic health and therapy development occurred in the stroke field. Stroke, the fifth 
leading cause of death in the USA, which attracted heavy investment by pharmaceutical companies in the 1990s, has very little therapy development remaining in industry. NINDS's mandate is to reduce the burden of disease due to neurological disorders and stroke. This mission is inherently tied to making better prevention, treatment and recovery therapies available to patients, and this occurs primarily through commercial development. Although industry can make bottom-line decisions to drop programs based on financial risk assessments, NINDS has to stay true to its commitment for all the disorders in its portfolio. Operationally, this means tying basic neuroscience to the neurobiology of disease, and the neurobiology of disease to the functional consequences of the disorder in patients.

NINDS-funded therapy development involves moving to industry those discoveries from the neurobiology of disease laboratories with the potential to prevent or improve the functional consequence of disease for patients. In addition, NINDS-funded investigators can revolutionize therapy development in a field by identifying and validating biomarkers related to the neuropathology or outcome measures that provide a pathway for testing new therapies. The link between the more common contrast-enhancing magnetic resonance imaging lesions with the less common clinical exacerbations of multiple sclerosis is a wonderful example of a biomarker that enabled development of a number of disease-modifying therapies. The direct NINDS investment in the therapy development space varies among disorders. The usual term for this investment is "de-risking", which may be minimal if industry is aggressively pursuing discovery-to-therapy for a disorder, or more substantial in cases in which Phase 1 studies in patients are necessary to engage commercial interest. An exception to the "de-risking" goal is the congressionally mandated small business grant program (3.3\% of the NINDS budget), which directly funds businesses, or collaborations between business and academia, to develop products for neurological health.

In 2002, NINDS began work to create a group of program directors and cooperative agreement mechanisms for funding therapy development across its portfolio, including the small business grant program [3]. Based on scientific and industry community input to the NINDS strategic planning process, this effort was incorporated into an Office of Translational Research with program staff and hands-on experience in therapy development, an experienced steering committee, and access to contracts for many of the processes required for therapy development.

\section{Programs of the NINDS Office of Translational Research}

The mission of the NINDS Office of Translational Research (OTR) is to accelerate the preclinical discovery and development of new therapeutic interventions for neurological disorders (http://www.ninds.nih.gov/funding/areas/ translational_research/). The OTR provides approximately $\$ 100$ million in funding and resources annually through grants, cooperative agreements, and contracts to academic and industry researchers to advance early-stage neurological technologies, devices, and therapeutic programs to industry adoption. The OTR comprises 6 programs that support the design, implementation, and management of research activities to critical translational challenges in neurology, outlined in the following subsections.

\section{The Anticonvulsant Screening Program}

The Anticonvulsant Screening Program (ASP; www.ninds. nih.gov/research/asp) has facilitated the discovery of new anticonvulsants for persons with epilepsy. For $>40$ years the ASP screened compounds for antiseizure activity in a battery of well-established rodent seizure models. Throughout its history, the ASP has played a role in the identification and development of numerous Food and Drug Administrationapproved antiepileptic drugs, including felbamate (Felbatol), topirimate (Topamax), lacosamide (Vimpat), and retigabine (Potiga). The focus of the ASP is now being redirected toward unmet medical needs in epilepsy, specifically as treatments for refractory epilepsies and the more difficult goal of testing therapies for their ability to modify the disease itself, so called antiepileptogenesis (the development of epilepsy in previously unaffected individuals and disease progression). A new resource, PANAChE (Public Access to Neuroactive and Anticonvulsant Chemical Evaluations; http://panache.ninds.nih. gov/) provides open access to an online database of nonproprietary chemical structures and biological data for compounds that have been screened through the ASP. Compounds submitted to ASP for testing are included only with the explicit permission of the supplier.

\section{The Cooperative Research to Enable and Advance Translational Enterprises Program}

The Cooperative Research to Enable and Advance Translational Enterprises (CREATE) program (www.ninds.nih.gov/ funding/CREATE) offers NIH funding through the cooperative agreement and Small Business Innovation Research (SBIR) Fast-Track award mechanisms. These are milestone-driven mechanisms supported by NINDS staff who help develop the project plan, monitor research progress, and make appropriate go/no-go decisions.

\section{The CREATE Bio Program}

The CREATE Bio program, established in July 2014, supports biotechnology product- and biologics-based therapies (e.g., peptides, proteins, oligonucleotides, gene therapies, and cell 
therapies) and lead optimization to early clinical trials (http:// www.ninds.nih.gov/funding/areas/translational_research/ CREATE-Bio.htm). This program emphasizes crossdisciplinary collaboration with an end goal in mind.

The CREATE Bio Discovery Track The CREATE Bio Discovery Track supports the optimization of therapeutic lead(s) showing convincing proof of concept. The end goals of the discovery track are to characterize and select a candidate that has bioactivity, stability, and bioavailability, as well as in vivo efficacy and/or target engagement that are consistent with the desired clinical application. At the end of the up to 4-year funding period, projects that successfully advance through this program will be ready for entry into the CREATE Bio Development track for further development to enable filing for an Investigational New Drug (IND) application.

\section{The CREATE Bio Development Track The CREATE Bio} Development Track supports IND-enabling studies and earlyphase clinical trials with the end goal of submitting an IND to the Food and Drug Administration. Funded for up to 5 years, this program supports early-phase clinical trials as an extension to the preclinical development programs, although these are not required components of proposed projects.

\section{The CREATE Devices Program The CREATE Devices} program supports the development, testing, and demonstration of therapeutic devices that treat neurological disorders (http://www.ninds.nih.gov/funding/areas/translational_ research/CREATE-Devices.htm). There are currently three tracks for devices:

1) Translational and Clinical Studies to Inform Final Device Design supports development of a device to test scientific hypotheses that are not feasible or practical to conduct in animal models but are critical to enable next-generation devices. Preclinical work supported by the program should lead to an Investigational Device Exemption (IDE) to support a clinical study, or a Non-Significant Risk study that does not require an IDE. Activities supported through this program include the implementation of clinical prototype devices, preclinical safety and efficacy testing, design verification and validation activities, pursuit of regulatory approval for the clinical study, and a clinical study.

2) Translational and Clinical Studies on the Path to $510(\mathrm{k})$ supports preclinical studies and the following IDEenabled Non-Significant Risk studies. It is expected that the immediate next steps upon completion of the clinical study will be a $510(\mathrm{k}) / 510(\mathrm{k})$ de novo submission or a larger clinical trial that will lead directly to a $510(\mathrm{k}) /$ $510(\mathrm{k})$ de novo submission. Activities supported through this program include implementation of clinical prototype devices, preclinical safety and efficacy testing, design verification and validation activities, pursuit of regulatory approval for the clinical study, and a clinical study.

3) Translational and Early Feasibility Studies on the Path to Premarket Approval (PMA) or Humanitarian Device Exemption supports applications to pursue preclinical studies for an IDE submission, with the option of also supporting an Early Feasibility Study. The next steps upon completion of the Early Feasibility Study should be a full Feasibility Study and a Pivotal Trial in support of a Premarket Approval or Humanitarian Device Exemption. Activities supported in this program include implementation of clinical prototype devices, preclinical safety and efficacy testing, design verification and validation activities, pursuit of regulatory approval for the clinical study, and an Early Feasibility Study.

\section{The Blueprint Neurotherapeutics Network}

The Blueprint Neurotherapeutics Network (BPN) provides support for small molecule drug discovery and development, from hit-to-lead chemistry through Phase I clinical testing (http://neuroscienceblueprint.nih.gov/bpdrugs/). The NIH Blueprint is a consortium of all the neuroscience-related Institutes and Centers, and BPN funds therapy development projects within each of those Institutes and Centers' missions. BPN offers NIH funding to neuroscience industry and academic investigators through the cooperative agreement and SBIR Fast-Track award mechanisms. In addition, BPN offers grantees access to a network of NIH-funded contract research organizations and consultants with extensive industry experience in the areas of assay development, pharmacology, medicinal chemistry, pharmacokinetics, toxicology, process research, chemical development, formulation development and Phase I clinical safety testing. Projects can enter either at the Discovery or Development stage, and are milestone-driven. Projects that enter at the Discovery stage will optimize well-validated hit compounds through medicinal chemistry to identify a development candidate. Projects at the Development stage will advance development candidates through IND-enabling toxicology studies and Phase I clinical testing. BPN participants receive intellectual property rights to drug candidates developed through the program. Since its inception, BPN has successfully launched 15 therapy development projects and built a nationwide virtual network of contractors and consultants with extensive drug discovery and development experience.

\section{The Innovation Grants to Nurture Initial Translational Efforts Program}

The Innovation Grants to Nurture Initial Translational Efforts (IGNITE) program enables grantees to build on their innovative basic science findings in advance of embarking on laborintensive therapy development activities as supported by the 
CREATE and BPN programs (http://www.ninds.nih.gov/ funding/areas/translational research/ignite.htm). Launched in December 2014, IGNITE is designed with 4 funding opportunities:

1) Assay Development and Therapeutic Agent Identification and Characterization to Support Therapeutic Discovery supports new in vitro and ex vivo assays and iterative screening efforts to identify and characterize novel therapeutic agents for neurological disorders.

2) Pharamcodynamics and in vivo Efficacy Studies for Small Molecules and Biologics/Biotechnology Products supports the implementation of pharmacodynamics, pharmacokinetics, and in vivo efficacy studies to demonstrate that proposed therapeutic agents are appropriately chosen for further development as a treatment for a neurological disorder.

3) Two additional IGNITE programs, Neuroscience Translational Tools, and Models and Neuroscience-focused Translational Platform Technology Development, are slated for release in mid-2015. These programs will focus on validating translatable animal models and quantifiable markers of target engagement applicable in humans, and neurosciencefocused translational platform technology development that enables and accelerates drug discovery for neurological disorders (e.g., overcoming blood-brain barrier permeability).

\section{The Countermeasures Against Chemical Threats (CounterACT) Program}

The Countermeasures Against Chemical Threats (CounterACT) Program is a translational research program that supports basic, translational, and clinical research aimed at the discovery or identification of better therapeutic medical countermeasures and/or diagnostic technologies against chemical threat agents (www.ninds.nih.gov/counteract). NIH leads the CounterACT program, which seeks to integrate cutting-edge basic, translational, and clinical research with the latest technological advances in science and medicine for a more rapid and effective response during chemical emergencies. CounterACT also facilitates the movement of medical countermeasures and diagnostic technologies through the regulatory process in collaboration with other federal departments and agencies, such as the Biomedical Advanced Research and Development Authority. This trans-NIH effort led by NINDS includes a comprehensive network of Research Centers of Excellence, individual cooperative research projects, SBIR grants, contracts, and interagency agreements with the Department of Defense.

\section{NINDS SBIR}

The NINDS SBIR funds small business concerns to conduct innovative neuroscience research at different stages of development, including applied bench research, translational research, and early-stage clinical trials. The NIH small business program began as a congressionally mandated set-aside program to encourage research and development leading to commercialization. It is intended to stimulate a partnership of ideas and technologies with the private sector and strengthen the role of small business in meeting federal research or research and development needs. The NINDS small business program has 2 components: 1) the SBIR program helps small business concerns to engage in research and development with the potential for commercialization; 2) the Small Business Technology Transfer Program facilitates cooperative research and development between small business concerns and US research institutions.

In fiscal year 2014, the NINDS made SBIR grant awards exceeding \$38 million and Small Business Technology Transfer grant awards exceeding $\$ 5.5$ million.

These early- and late-stage therapy development funding programs are designed to accelerate the preclinical discovery and development of new therapeutic interventions for neurological disorders. NINDS encourages grantees to expand their expertise base and improve the quality of their team through multidisciplinary research. Additionally, the NINDS OTR recently issued a new suite of funding opportunities to further prime the pump for neurotherapeutics [4]. However, we recognize that key outstanding needs remain in the field of translational neuroscience. Better understanding of basic disease mechanisms combined with an improved ability to translate such discoveries into biomarkers and therapeutics will accelerate progress toward better treatments and, ultimately, cures for neurological disease. The goal of these programs is to build a bridge connecting basic science to industry that accelerates the advancement of promising projects to clinical testing in order to more quickly benefit patients suffering from neurological disorders.

Required Author Forms Disclosure forms provided by the authors are available with the online version of this article.

\section{References}

1. Koroshetz W and Landis S. Neurology's stake in foundational neuroscience research. JAMA Neurol 2014;71:1081-1082.

2. Pankevich DE, Altevogt BM, Dunlop J, Gage FH, Hyman SE. Improving and accelerating drug development for nervous system disorders. Neuron 2014;84:546-553.

3. Finkelstein R, Miller T, Baughman R. The challenge of translational research - a perspective from the NINDS. Nat Neurosci 2002;5: 1029-1030.

4. Ranganathan, R. NINDS translational programs: priming the pump of neurotherapeutics discovery and development. Neuron 2014;84: 515-520. 